\title{
Critical parameters of $N$-vector spin models on 3d lattices from high temperature series extended to order $\beta^{21 *}$
}

\author{
P. Butera and M. Comi ${ }^{\text {a }}$

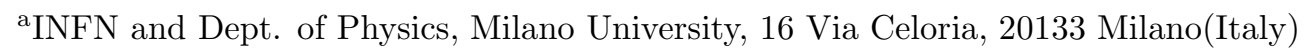

\begin{abstract}
High temperature expansions for the free energy, the susceptibility and the second correlation moment of the classical $N$-vector model [also denoted as the $O(N)$ symmetric classical spin Heisenberg model or as the lattice $O(N)$ nonlinear sigma model] have been extended to order $\beta^{21}$ on the simple cubic and the body centered cubic lattices, for arbitrary $N$. The series for the second field derivative of the susceptibility has been extended to order $\beta^{17}$. An analysis of the newly computed series yields updated estimates of the model's critical parameters in good agreement with present renormalization group estimates.
\end{abstract}

\section{Introduction}

We have extended to order $\beta^{21}$ the computation of the High Temperature (HT) series for the free energy, the susceptibility $\chi(\beta, N)$, the second correlation moment $\mu_{2}(\beta, N)$ and the second field derivative of the susceptibility $\chi_{4}(\beta, N)$ for the classical $N$-vector model with any $N$ and on all bipartite lattices, e.g. the (hyper) simple cubic (sc) and (hyper) body centered cubic (bcc). The HT coefficients are written as explicit (rational) functions of $N$ and it is also possible to exhibit their (polynomial) dependence on the space dimensionality $d$.

The model Hamiltonian is

$$
H\{v\}=-1 / 2 \sum_{\left\langle\vec{x}, \vec{x}^{\prime}\right\rangle} v(\vec{x}) \cdot v\left(\vec{x}^{\prime}\right)
$$

where $v(\vec{x})$ is a $N$-component classical unit spin. As well known, for $N=0$ we get the selfavoiding walk model, for $N=1$ the Ising $S=1 / 2$ model, for $N=2$ the XY model, for $N=3$ the classical Heisenberg model, for $N \rightarrow \infty$ the (exactly solvable) spherical model. We have used the vertex-renormalized Linked Cluster Expansion (LCE) method. Due to lack of space we address the reader to Refs. 11 14 for an extensive bibliography on the LCE technique and on the HT series published and analyzed before our work. Here we shall only quote the paper by M. Lüscher

\footnotetext{
*Presented by P. Butera
}

and P.Weisz [5] who computed HT series by LCE through $\beta^{14}$ for $\chi, \mu_{2}$ and $\chi_{4}$ on the sc lattices in $d=2,3,4$. Our work has been made possible by an extensive redesign of the algorithms proposed in Ref. [5] in order to reduce drastically the growth of the computational weight with the order of the expansion. It is well known that the complexity of the HT expansions grows exponentially with the order so that obtaining one more series coefficient requires an effort substantially larger than for all previous ones. Let us now sketch the calculation of $\chi$ through order $\beta^{L}$. First we have to list all topologically inequivalent multigraphs of the class $S_{2}(L)$ defined as follows: i) they have 2 external lines and no more than L internal lines; ii) they are connected, 1-vertex, 1-line irreducible, and contain no odd loops; iii) they have only even degree vertices. The Feynman rules are: for each graph $G \in S_{2}(L)$ we have to compute

$$
F(G ; N ; d ; \beta)=\frac{I(G, d) C(G, N) W(G, N, d, \beta)}{S(G)}
$$

where $S(G)$ is the symmetry number of the graph $G$ (an integer number depending only on the structure of $G) ; C(G, N)$ is the group coefficient of $G$, related to the $O(N)$ symmetry group of the model (a polynomial in $N$ with integer coefficients); $I(G, d)$ is an integer number counting the unrestricted lattice per-site embeddings of $G$ (it depends on $G$ and the lattice structure and 
dimensionality $d) ; W(G, N, d, \beta)$ is associated to the vertices of $G$ : it depends on the structure of the spin interaction and accounts ("renormalization") for all vertex insertions in the graphs $\in S_{2}(L)$ (it is a polynomial in $\beta$ and $d$ and a rational function of $N)$. Then we sum over all $F(G ; N ; d ; \beta)$

$$
\chi(N, \beta)_{1 L I}=\sum_{G \in S_{2}(L)} F(G ; N ; d ; \beta)
$$

and finally

$$
\chi(N, \beta)=\frac{\chi(N, \beta)_{1 L I}}{1-4 d \beta \chi(N, \beta)_{1 L I}}
$$

It is now clear that in order to get long series we need fast and efficient combinatorial algorithms for producing and comparing graphs, since the sum extends to all topologically inequivalent graphs in $S_{2}(L)$. But generation algorithms inevitably produce duplicate graphs which must be discarded and identification algorithms have a computational weight exponentially increasing with the number of vertices! Fast algorithms are also needed to compute the symmetry number $S(G)$ of each graph: this is also a task of exponential complexity. Moreover the computational load of the algorithm for the embedding number $I(G, d)$ grows fastly with $d$.

The intricacies and the size of this calculation can be gauged from the number of graphs $\approx 2 \cdot 10^{7}$, which enter into the computation of $\chi$ through order $L=21$. This figure should be compared with the corresponding one $\approx 7 \cdot 10^{3}$ of the $\mathrm{O}\left(\beta^{14}\right)$ computation in Ref. [5]. However we are still far from our present computational limits!

It is also worth to stress that the LCE gives immediate access also to series for the more general model described by the partition function:

$$
Z=\int \Pi d \mu\left(\vec{\varphi}_{i}^{2}\right) \exp \left[\beta \sum_{\langle i, j\rangle} \vec{\varphi}_{i} \cdot \vec{\varphi}_{j}\right]
$$

where $\vec{\varphi}_{i}$ is a $N$-component vector. By a proper choice of the single spin measure $d \mu\left(\vec{\varphi}_{i}^{2}\right)$ we obtain the HT series for a variety of models including the general scalar isovector $P\left(\vec{\varphi}^{2}\right)$ lattice field theory, the general spin $S$ Ising model, the BlumeCapel model, the double Gaussian model..., and this enables us to study various representatives of each universality class. The expected returns of our laborious enterprise include more accurate universality tests, comparisons with estimates of critical exponents and of critical amplitude ratios obtained within the Renormalization Group (RG) approach [6] either by the Fisher-Wilson perturbative $\epsilon$-expansion $(\epsilon=4-d)$ of the continuum $\left(\vec{\varphi}^{2}\right)^{2}$ model, or by the Parisi coupling constant expansion of the same model in fixed dimension $d=3$, or else by other numerical methods. We also expect that the new feature of our calculation, namely the explicit dependence on $N$ and $d$ of our HT coefficients, can provide further insight into the properties of $1 / N$ and $\epsilon$ expansions.

This project has been carried on by an IBM Risc $6000 / 580$ w-station with 128 Mbytes memory and 1.5 Gbytes disk storage. Typical cpu times are a few hours.

\section{Series Analysis}

Our HT series on the square lattice have been tabulated and examined in Refs. [3.44], while a study of the $3 \mathrm{~d}$ case has been partly presented in Refs. [1].2]. In the $2 \mathrm{~d}$ case, our results can be summarized as follows: the HT series exhibit in the range $N \leq 2$ a qualitative behavior which is sharply different from that for $N>2$. For $N=2$ the critical properties of the model are completely consistent with the current ideas on the Kosterlitz-Thouless phase transition. For $N>2$ they appear to be consistent with the conventional asymptotic freedom expectations from the perturbative RG analysis and with exact (although not completely rigorous) results from the Bethe Ansatz. Similar conclusions from an independent computation of $2 \mathrm{~d}$ HT expansions have been reached in Ref. [7]. In the $3 \mathrm{~d}$ case, we have a more conventional scenario: for all $N$ a second order phase transition occurs at nonzero temperature. In particular, if we set $\tau \equiv 1-\beta / \beta_{c}$, in the vicinity of the critical point $\chi$ and $\xi$ behave as

$$
\begin{gathered}
\chi(N, \beta) \simeq A_{\chi}(N) \tau^{-\gamma(N)}\left(1+a_{\chi}(N) \tau^{\theta(N)}+\right. \\
\left.a_{\chi}^{\prime}(N) \tau^{2 \theta(N)}+. .+e_{\chi}(N) \tau+. .\right)
\end{gathered}
$$


and

$$
\begin{gathered}
\xi(N, \beta) \simeq A_{\xi}(N) \tau^{-\nu(N)}\left(1+a_{\xi}(N) \tau^{\theta(N)}+\right. \\
\left.a_{\xi}^{\prime}(N) \tau^{2 \theta(N)}+. .+e_{\xi}(N) \tau+. .\right)
\end{gathered}
$$

when $\tau \downarrow 0$. The critical exponents $\gamma(N), \nu(N)$ and the scaling correction exponent $\theta(N)$ are universal (for each $N$ ). On the contrary the critical amplitudes $A_{\chi}(N), a_{\chi}(N), . ., A_{\xi}(N), a_{\xi}(N)$.. are nonuniversal, but amplitude ratios like $a_{\xi}(N) / a_{\chi}(N)$ etc. are universal and interesting since they are not yet accurately known.

The numerical problem of determining simultaneously the critical parameters $\beta_{c}(N), \gamma(N)$, $\nu(N)$ etc. as well as the leading correction exponent $\theta(N)$ is a difficult job: it amounts to an intrinsically unstable double exponential fit. However it must be faced, because without proper allowance at least for the main corrections to scaling no improvement of the accuracy in the determination of critical parameters can be warranted even from extended series. We have therefore performed two kinds of analysis of the series by the differential approximant method:

i) an unbiased analysis, where we do not assume to know some critical parameters when trying to determine the remaining ones;

ii) a biased analysis, where we assume that the exponents of the confluent corrections to scaling, take the values indicated by the fixed dimension RG perturbative calculations in Ref. [6] for $N \leq 3$ and in Ref.[9] for $N>3$.

From the analysis of $\chi$ and $\xi$, we obtain in this way numerical estimates of critical parameters which are shown in two extensive tables of Ref.[1], but cannot be reported here for lack of space. We can however summarize the qualitative conclusions. As a first general result, both analyses confirm the traditional expectation that the bcc series have much better convergence properties than the sc series. The results of the unbiased series analysis agree well with the exponent estimates from the fifth order $\epsilon$-expansion over the range $0 \leq N \leq 3$ on which they are available. Analogously, we observe an even closer agreement with the results from the fixed dimension coupling constant six loop expansion [6, 10] over the whole range of values of $N$, and, for $0 \leq N \leq 3$, with the seven loop results 11. However for $N \geq 4$, the presence of residual trends in the extrapolations of the series estimates for the exponents which use an increasing number of HT coefficients, seriously questions the accuracy of the unbiased analysis suggesting that the confluent corrections are large and inadequately accounted for. Therefore a biased analysis is necessary.

From the biased analysis we also obtain interesting and, in some cases, accurate estimates of various critical amplitudes which confirm that scaling corrections should not be neglected. For $N \leq 3$, our biased exponent estimates essentially agree with the unbiased ones, but they have a greater accuracy. For $N \geq 4$, due to the large confluent corrections, the biased estimates of the critical exponents differ up to a few percents both from the unbiased ones and from the fixed dimension six loop perturbation results, suggesting that both a seven loop computation and a more accurate evaluation of the renormalized coupling may be needed.

\section{REFERENCES}

1. P.Butera and M.Comi, Phys. Rev. B56 (1997) 8212 (hep-lat/9703018).

2. P.Butera and M.Comi, Phys. Rev. B52 (1995) 6185.

3. P.Butera and M.Comi, Phys. Rev. B54 (1996) 15828.

4. P.Butera and M.Comi, Phys. Rev. B47 (1993) 11969.

5. M. Lüscher and P.Weisz, Nucl. Phys. B300 (1988) 325.

6. J. Zinn Justin, Quantum field theory and critical phenomena (Clarendon, Oxford,1996).

7. M. Campostrini,A. Pelissetto, P.Rossi, E. Vicari, Phys. Rev. D54 (1996) 1782.

8. A. J. Guttmann, in Phase Transitions and critical Phenomena, edited by C. Domb and J. Lebowitz, (Academic, New York, 1989) Vol. 13.

9. A. I. Sokolov, unpublished.

10. S. A. Antonenko and A. I. Sokolov, Phys. Rev. E51 (1995) 1894.

11. D. B. Murray and B. G. Nickel, Unpublished Guelph University report (1991). 